\title{
Down-regulation of let-7a and miR-21 in urine exosomes from lupus nephritis patients during disease flare
}

\author{
Pattarin Tangtanatakul, ${ }^{1,2}$ Siriwan Klinchanhom, ${ }^{2,3}$ Pimpayao Sodsai, ${ }^{2,3}$ Thitima Sutichet,,$^{2,4}$ Chutipha Promjeen, ${ }^{2,4}$ \\ Yingyos Avihingsanon, ${ }^{2,4}$ Nattiya Hirankarn ${ }^{2,3 *}$
}

\begin{abstract}
Background: The contents of exosomes determine their biological functions and represent a new class of epigenetic modulation of renal cells considering as novel class of biomarker.

Objective: Our study aims to investigate the expression of microRNAs (miRNA), including miR-10a/b, let-7a, and miR-21, in urinary exosomes isolated from patients with active lupus nephritis (LN) compared to inactive LN.

Methods: The exosomes were obtained from long-term follow up LN patients during active and 4 months after treatment $(\mathrm{n}=3)$. The expression of candidate miRNAs was validated in group of LN patients with renal flare $(\mathrm{n}=13)$ and remission stage $(n=18)$ using qPCR. All exosomes were characterized by NanoSight and western blotting. The correlation between miRNA expressions and kidney functions was studied.

Results: We found that let-7a and miR-21, but not miR-10a/b, were significantly down-regulated in LN patients with active disease compared with inactive disease. Long-term follow-up patients also showed down-regulation of let-7a and miR-21 during disease flare while the expressions were elevated after complete course of treatment. Although the miRNA expressions were not significantly correlated with classical kidney injury markers, negative correlations were found in both protein leakages and glomerular filtration rate.

Conclusion: Our result suggested that urinary exosome-associated miRNA, let-7a and miR-21, could be used to guide the clinical stage of LN patients and possibly plays a role in epigenetic regulation of the kidney during the disease. Its expression might be able to use as liquid biopsy, however, validation in large sample size is required to show it significant in clinical implication.
\end{abstract}

Key words: miRNA, Urinary exosomes, Lupus Nephritis, let-7a, miR-21

\section{From:}

Medical Microbiology Interdisciplinary Program, Graduate School, Chulalongkorn University, 10330, Thailand

${ }^{2}$ Center of Excellence in Immunology and Immune-mediated Disease, Faculty of Medicine, Chulalongkorn University, 10330, Thailand

Department of Microbiology, Faculty of Medicine, Chulalongkorn University, 10330, Thailand

${ }^{4}$ Department of Medicine, Faculty of Medicine, Chulalongkorn University, 10330, Thailand

\section{Introduction}

Lupus Nephritis (LN) is characterized by autoantibodyinduced renal damage in patients with Systemic Lupus Erythematosus (SLE). Disease immunopathogenesis is driven by the deposition of immune-complexes in the kidney, inducing lysis of intrinsic renal cells and inflammation. ${ }^{1}$ High doses of immunosuppressant is applied to control disease in patients with severe LN; however, some LN patients fail to respond to

\author{
* Corresponding author: \\ Nattiya Hirankarn \\ Department of Microbiology, Faculty of Medicine, \\ Chulalongkorn University, 10330, Thailand \\ Email: Nattiya.H@chula.ac.th
}

treatment and cannot achieve remission stage. ${ }^{2}$ At present, renal tissue biopsy is the gold standard to directly evaluate injury to the kidney and determine the proper course of treatment. ${ }^{3}$ However, it is an invasive procedure that puts the patients at risk of bleeding or infection. ${ }^{4}$ Therefore, a non-invasive biomarker is urgently needed to easily monitor disease progression and to predict diseases recurrence within the kidney. 
Extracellular vesicles (EVs) are small vesicles (size range between $40 \mathrm{~nm}$ and $2 \mu \mathrm{m}$ ) secreted by various types of cells and can be found in several body fluids including cerebrospinal fluid, synovial fluid, saliva, serum, and urine. ${ }^{5}$ EV s can be classified by their biogenesis and related functions into three main classes: exosomes, microvesicles (shedding vesicles), and apoptotic bodies. ${ }^{6}$ Interestingly, recent study demonstrated that exosomes could contain several subcellular organelles, mitochondrial components, and extracellular receptors that might be important in immune mediated such as MHC-class II with co-stimulatory molecules. ${ }^{7}$ Moreover, various reports showed that exosomes deliver messenger RNA (mRNA) and microRNA (miRNA) to regulate gene and protein expression in recipient cells. ${ }^{8}$ This is a relatively novel mechanism in term of epigenetic regulation and long-distance transportation of molecules. ${ }^{9}$ Therefore, exosomes have been hypothesized to be important biomarkers due to the discovery of their physiological role in cell-to-cell communication, as well as their potential to be used as a non-invasive liquid biopsy tool. ${ }^{10}$

The miRNAs are small non-coding RNA which block protein translation and induce mRNA degradation. ${ }^{11}$ Experiment has shown that miRNAs are highly stable within exosomes and could act on recipient cells after being recognized by receptors or undergoing endocytosis. ${ }^{12}$ Urinary exosome -associated miRNAs play an important role as epigenetic factors maintaining renal tubular cells in a paracrine manner. ${ }^{13}$ In patients with LN, a subset of miRNAs (miR-335*, miR-200c, miR-302d and miR-146a) within urinary exosomes, but not miRNAs from cell-free urine or urine supernatant, were increased in patients with active LN compared with healthy controls. ${ }^{14}$ Puromycin-induced injury of immortalized mouse podocytes increased the release of exosomes containing miR-26a. ${ }^{15}$ Furthermore, the level of miR-29c in exosomes was found to specifically correlate with fibrosis in LN patients compared with other chronic kidney diseases. ${ }^{16}$ Although these evidences highlighted the important of urinary exosomes containing miRNA which could reflect the renal histopathological changes in LN patients, a marker to predict disease relapse or therapeutic achievement is still unknown.

Interestingly, the study in spontaneous develop LN mice revealed disease associated miRNA induced by immunosuppressant treatment. ${ }^{17}$ The study found that urinary miR-21 were decreased after hydroxychloroquine (HCQ) and prednisolone (PRED) treatment. ${ }^{18}$ The miR-21 was reported to control several key inflammatory molecules such as IFN- $\gamma$ and IL-12 which is associated with autoimmune disease development. ${ }^{19}$ Furthermore, the urinary miR-21 was identified as a promising biomarker for kidney injuries and fibrosis..$^{20,21}$ As miRNA were highly stable in urinary exosomes, we therefore suspected that miR-21-associated urinary exosomes might be a candidate marker to determine disease relapse or treatment response in LN patients. Moreover, according to our previous study, we found that miR-10a, miR-10b and let-7a expression was rapidly changed during acute immune complexes attack resident renal cells, ${ }^{22}$ it is possible that abnormal miRNA expression might be secreted through urinary exosome and act as a detection marker for disease recurrence and treatment responses. In addition, abnormal miR-10a, miR-10b and let-7a expression could reflex the inflammatory phenotypes of resident kidney cells. ${ }^{23}$ For example, the miR-10a down-regulated IL-8 expression which is a leukocyte chemoattractant during acute inflammation as well as the let-7a regulated IL-6 expression..$^{22,24}$ Thus, detecting miR-10a, miR-10b and let-7a associated urinary exosomes might be a new potential biomarker for LN patients. The objective of this study is to characterize miR-21, miR-10a, $\mathrm{miR}-10 \mathrm{~b}$, and let-7a expression in urinary exosomes during course of LN disease. The urinary exosome-associated miRNAs might be able to serve as a non-invasive biomarker, as well as help provide insight into novel epigenetic modulation during the onset of LN or in ongoing disease.

\section{Methods \\ Samples Collection}

This study was conducted under Faculty of Medicine Research Ethics Committee approval (EC-136/59) with informed consent of all participants. All LN patients fulfilled at least four of eleven criteria from the Systemic Lupus Erythematosus Disease Activity Index (SLEDAI) diagnosis for SLE and had a kidney biopsy-proven LN histology classification. ${ }^{25}$ Random urines $(12 \mathrm{ml}$ ) were collected from $31 \mathrm{LN}$ patients (13 with active disease and 18 with inactive disease). Demographic data are shown in Table 1. The inclusion criteria for active LN included (A) the presence of proteinuria greater than $1 \mathrm{mg}$ per day, and (B) the presence of white blood cells (WBC) or red blood cells (RBC) in urinalysis of greater than 5 cells per high power field or the presence of cellular casts without any urinary tract infection. The presence of WBC or RBC less than 5 cells per high power field was the inclusion criteria for inactive LN, without considering urine protein leakage. Additionally, we were able to collect three urine samples from the same LN patients while they had active disease prior to treatment and when reaching the inactive stage after 3-4 months of treatment. All samples were stored at $-20^{\circ} \mathrm{C}$ until used.

\section{Exosomes Isolation}

The urinary exosomes were isolated using a previous published protocol. ${ }^{26}$ In brief, urine $(12 \mathrm{~mL})$ was firstly centrifuge at $2,000 \times g$, for 10 minutes at $4^{\circ} \mathrm{C}(\mathrm{SX} 4750 \mathrm{~A}$, swing bucket rotor, Allegra X15R, Beckman, IN, USA) to remove cell debris and urinary cells. At this step, the pellet was collected for western blotting to check exosome marker. Subsequently, the supernatant was continuously centrifuged at $17,000 \times g$ for 45 minutes at $4^{\circ} \mathrm{C}$ (JA-14, fixed angle rotor, Avanti, Backman) to dispose of large molecular weight molecules contamination. After centrifugation, the supernatant $(1 \mathrm{~mL})$ was collected to represent as a urine supernatant for western blotting. The residual supernatants $(11 \mathrm{~mL})$ were carefully transferred to ultracentrifuge tube for urinary exosome isolation at 200,000 $\times g$ for 1 hour at $4^{\circ} \mathrm{C}$ (Ti-100 or $\mathrm{Ti}-45$, fixed angle rotor, Optima XPN-100, Beckman). After this step, the supernatant was discarded, and the pellet was defined as urinary exosomes. The exosomes were resuspended in $\mathrm{PBS}$ buffer, $\mathrm{pH} 7.4$ and stored at $-80^{\circ} \mathrm{C}$ until use.

\section{Western Blotting}

Components of ESCRT-1 complexes (Endosomal Sorting Complexes required for Transport-1) are required for vesicular trafficking processes and are important in exosomes biogenesis. Tetraspanin transmembrane proteins are also important 
Table 1. Demographic and clinical characteristics of the lupus nephritis patients.

\begin{tabular}{|c|c|c|c|}
\hline & $\begin{array}{l}\text { Active LN } \\
(n=13)\end{array}$ & $\begin{array}{l}\text { Inactive LN } \\
\quad(n=18)\end{array}$ & $P$-value \\
\hline Age, years ${ }^{*}$ & $32.77 \pm 9.02$ & $39.65 \pm 10.21$ & \\
\hline Gender, n [Female/Male] & $12 / 1$ & $18 / 0$ & \\
\hline Chronicity index & $1.5 \pm 1.9$ & $2.0 \pm 2.7$ & \\
\hline Activity index & $11.25 \pm 3.2$ & $10.4 \pm 3.3$ & \\
\hline \multicolumn{4}{|l|}{ Kidney Biopsy ${ }^{A}$} \\
\hline - Class III & 4 & 1 & \\
\hline - Class IV & 4 & 7 & \\
\hline - Class V+III & 2 & 3 & \\
\hline - Class V+IV & 1 & 4 & \\
\hline - Class III+IV & 1 & 1 & \\
\hline - Class V & 1 & 2 & \\
\hline \multicolumn{4}{|l|}{ Clinical Characteristic } \\
\hline - $\mathrm{sCr},{ }^{\mathrm{a}} \mathrm{mg} / \mathrm{dL}$ & $1.03 \pm 0.4$ & $0.97 \pm 0.66$ & \\
\hline - $\mathrm{UPCI}^{\mathrm{b}}$ & $1.37 \pm 0.77$ & $1.22 \pm 1.22$ & \\
\hline - $\mathrm{MDRD}^{\mathrm{GFR}}{ }^{\mathrm{c}}$ & $80.44 \pm 22.2$ & $92.8 \pm 24.1$ & \\
\hline - WBC, ${ }^{\mathrm{d}}$ cells/HPFs & $8.0 \pm 6.0$ & $1.4 \pm 0.9$ & $p<0.001$ \\
\hline - RBC, ${ }^{\mathrm{e}}$ cells/HPFs & $8.0 \pm 7.7$ & $1.2 \pm 0.8$ & $p<0.05$ \\
\hline
\end{tabular}

* All values are the mean \pm S.D. unless otherwise indicated. Abbreviations: ${ }^{\text {a }} \mathrm{sCr}$, serum creatinine; ${ }^{b}$ UPCI, urine protein creatine index; ${ }^{c}$ MDRD GFR, glomerular filtration rate as determined by the Modification of Diet in Renal Disease (MDRD) study equation; ${ }^{\mathrm{d}} \mathrm{WBC}$, white blood cells; ${ }^{\mathrm{e}} \mathrm{RBC}$, red blood cells.

${ }^{A}$ Kidney Biopsy is categorized based on the criteria from International Society of Nephrology/Renal Pathology Society (ISN/RPS) classification of lupus nephritis (2003).

in exosome generation. To confirm the presence of exosomes, we detected TSG101, a $50 \mathrm{kDa}$ ESCRT protein (1:1000 rabbit anti-human TSG101, clone 4A10, Abcam, Cambridge, MA, USA) and CD9, a $22 \mathrm{kDa}$ tetraspanin (1:2000 mouse anti-human CD9, clone EPRP2949, Abcam) by western blot. Western blotting was carried out using $100 \mu \mathrm{g}$ of protein from whole urine, urinary cell pellet, urinary supernatant, and exosomes, loaded on a $10 \%$ polyacrylamide gel and transferred to nitrocellulose membrane at $100 \mathrm{~V}$ for 1 hour. The secondary antibodies used were HRP-conjugated mouse anti-rabbit IgG and HRP-conjugated rabbit anti-mouse IgG (SC-2357, Santa Cruz Biotechnology, Dallas, TX, USA). The signals were developed using Super Signal West Femto substrate (Thermo Fisher Scientific, Bangkok, Thailand) and analyzed with Image Quant (GE Healthcare, Bangkok, Thailand).

\section{Transmission Electron Microscope}

The exosomes pellets were fixed in phosphate buffer containing 2\% paraformaldehyde and loaded on Formvar/carbon coated electron microscope grids (TED PELLA, California, The United States of America). The exosomes were viewed with a JEOL 1011 CX-T electron microscope (JEOL Ltd., Tokyo, Japan) operating at $200 \mathrm{Kv}$.

\section{Analysis of Urinary Exosomes Size by NanoSight}

The suspended urinary exosomes were diluted 1:50 in PBS, pH 7.4 buffer and analyzed on a NanoSight NS300 (405 nm laser diode) in the light scatter mode for quantification and scatter distribution according to the manufacturer's protocol (Malvern Instrument Inc., Malvern, UK). The threshold for tracking systems was set at five and analysis was performed around 30 seconds five times.

\section{The miRNA Isolation from Urinary Exosomes}

Total RNA was isolated from urinary exosomes using miRNeasy mini kit for serum/plasma according to the manufacturer's instruction (QIAGEN, Hilden, Germany). Briefly, the suspended urinary exosomes $(100 \mu \mathrm{L})$ were lysed using Qiazol lysis reagent $(600 \mu \mathrm{L})$ and three $\mu \mathrm{L}$ Cel-39 spike-in controls $\left(1.6 \times 10^{8}\right.$ copies/ $\mu \mathrm{L}$, Qiagen, Hilden, Germany) were added. Then, 1-bromo-3-chloropropane was added to separate out the RNA. The aqueous phase was applied onto miRNeasy mini columns then the total RNA was eluted using $14 \mu \mathrm{L}$ RNase -free water. The RNA concentration was determined using a NanoDrop (ND-1000, Thermo Fisher Scientific). The RNA samples with A260/280 ratio higher than 1.8 were used in downstream experiments.

\section{RT-PCR and Real-Time PCR}

The candidate miRNAs were reverse-transcribed and amplified using specific primers for miR-21 (assay ID 000397), miR-10b (assay ID 002218), let-7a (assay-ID 000377), and cel -39 (assay-ID 000200) from Thermo Fisher Scientific. During reverse transcription, a total of $10 \mathrm{ng}$ RNA was used to convert miRNA to cDNA using TaqMan reverse transcription kit (Applied Biosystems, Thermo Fisher Scientific). The qPCR was conducted with an Applied Biosystem ${ }^{\circledR} 7500$ Real-Time PCR System.

\section{Statistical Analysis}

Unpaired $t$-tests and Mann-Whitney $\mathrm{U}$ tests were used for comparisons between samples with normal and non-normal distributions, respectively. The relationship between miRNA expression and other urinary biomarkers was determined by Pearson's correlation and linear regression. P-values less than 0.05 were considered statistically significant. In vitro experiments were performed at least three times.

\section{Results}

In accordance with guidelines from the International Society of Extracellular Vesicles, ${ }^{26}$ the size and specific protein markers, in both membrane and cytosolic fractions, were characterized in the isolated urinary exosomes. We found that the isolated urinary exosomes particle sizes were ranged from 40 to $200 \mathrm{~nm}$ (Figure 1A). Exosome specific protein markers, the ESCRT protein (TSG101) and tetraspanin (CD9), were enriched in the exosome fraction but could not be detected in the cell pellet, urine supernatant or whole urine of patients with LN (Figure 1B). These results confirmed that exosomes had been successfully recovered after all the processes.

We firstly obtained samples from three LN patients at twotime points (before and 4 months after treatment). All three patients responded well to treatment and were defined as quiescent LN after treatment. Candidate miRNAs, including let-7a, miR-10a/b and miR-21, were investigated in urinary exosomes. Interestingly, we found let-7a and miR-21 were down-regulated in all patients during renal flare (Figure 2A), while miR-10a/b showed inconsistent results among patients. We further studied the expression of all candidate miRNA 

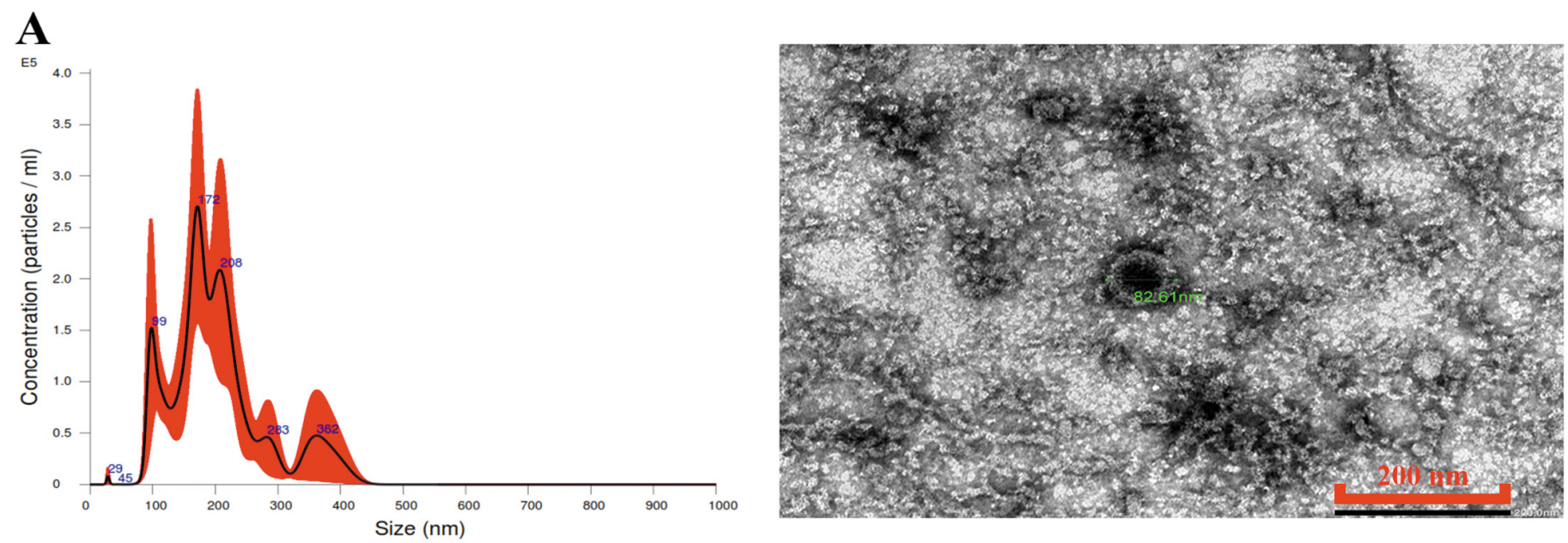

B

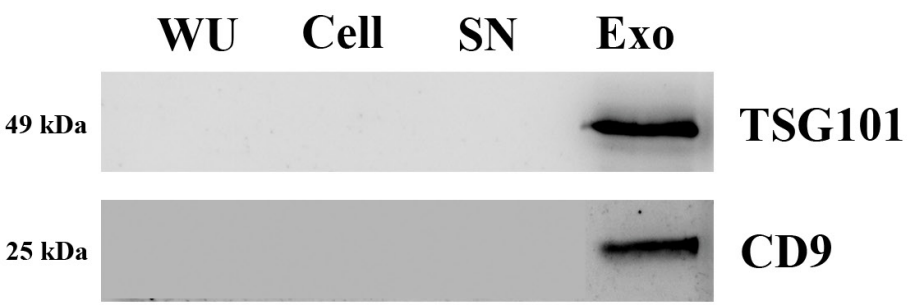

Figure 1. Characterization of urinary exosomes.

Urinary exosomes were obtained by ultracentrifugation at $200,000 \times g$ for 1 hour at $4^{\circ} \mathrm{C}$. (A) The graph shows the size of particles ranged from 40 to $200 \mathrm{~nm}$ (left). The presence of urinary exosomes was confirmed by transmission electron microscopy (right). The scale bar is $200 \mathrm{~nm}$. (B) Western blot showing enrichment of the exosome protein markers TSG101 and CD9 within urinary exosomes (Exo) compared with whole urine (WU), cell pellet (Cell), and urine supernatant (SN) from patients with LN.

A
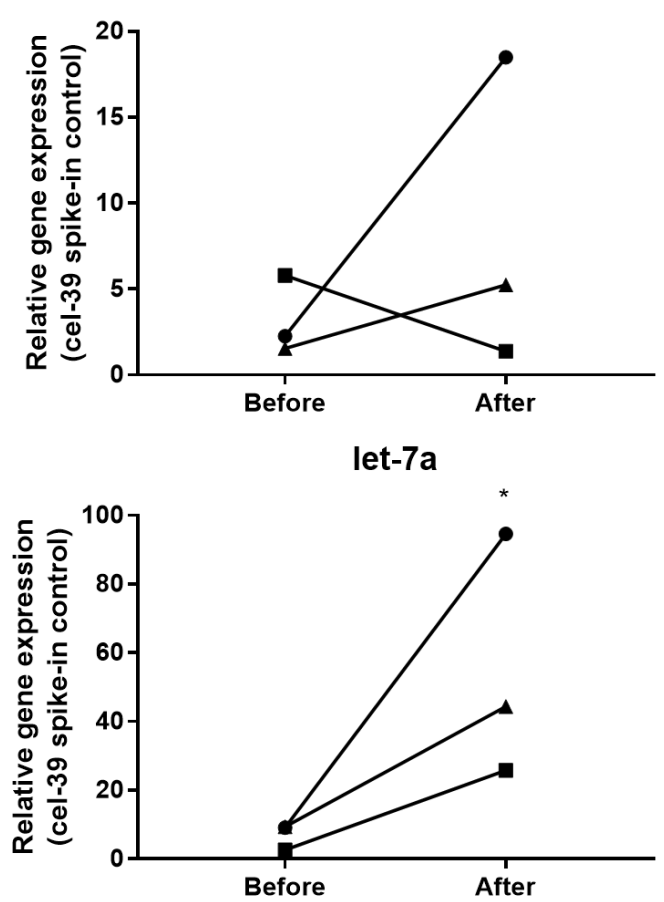

miR-10b
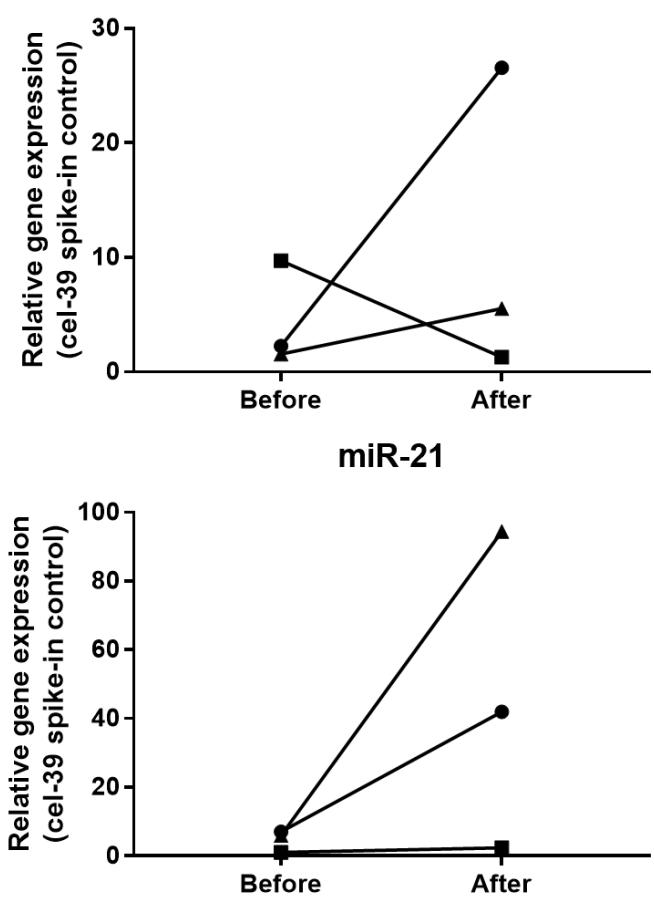

Figure 2. Validation and expression of urinary exosome-associated miRNA in patients with active and inactive LN.

A) Urinary exosomes were collected from patients before and after treatment $(n=3)$. The expression of miRNAs was detected using qPCR. The plots represent relative fold change of specific miRNA expression to cel-39 spike-in control in each patient compared to active $\mathrm{LN}$. 
B
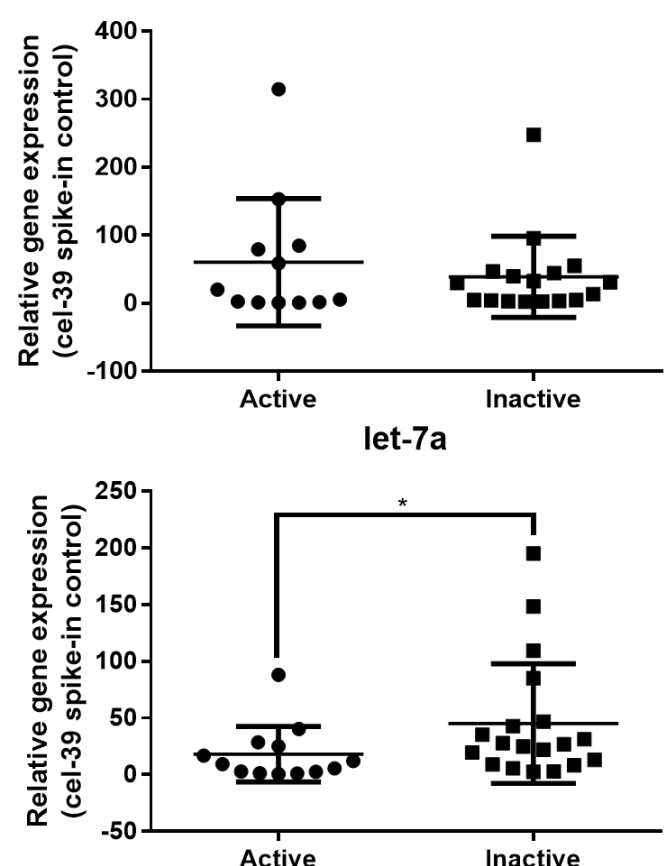

miR-10b

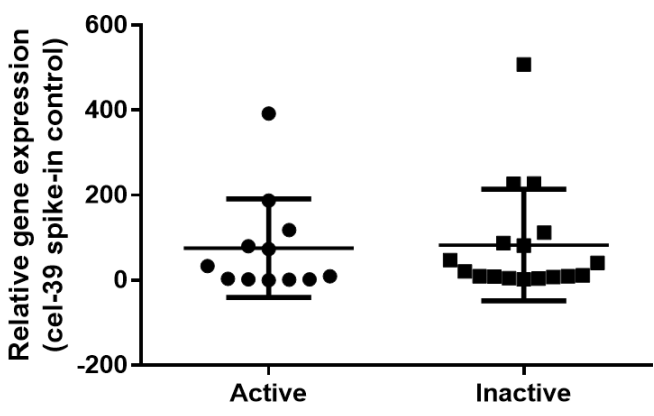

miR-21

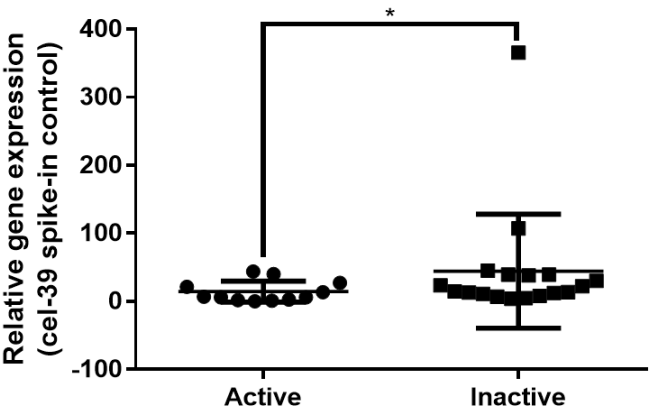

Figure 2. (Continued)

B) The graphs show selected miRNAs in patients with active $(n=13)$ and inactive $L N(n=18)$. The graphs were calculated from three replicate experiments and normalized with a spike-in control (cel-39). The differential expression of miRNA was compared using unpaired $t$-tests. The plots show the means and standard deviation. ${ }^{\star} p<0.05$.

Urine Protein Leakage
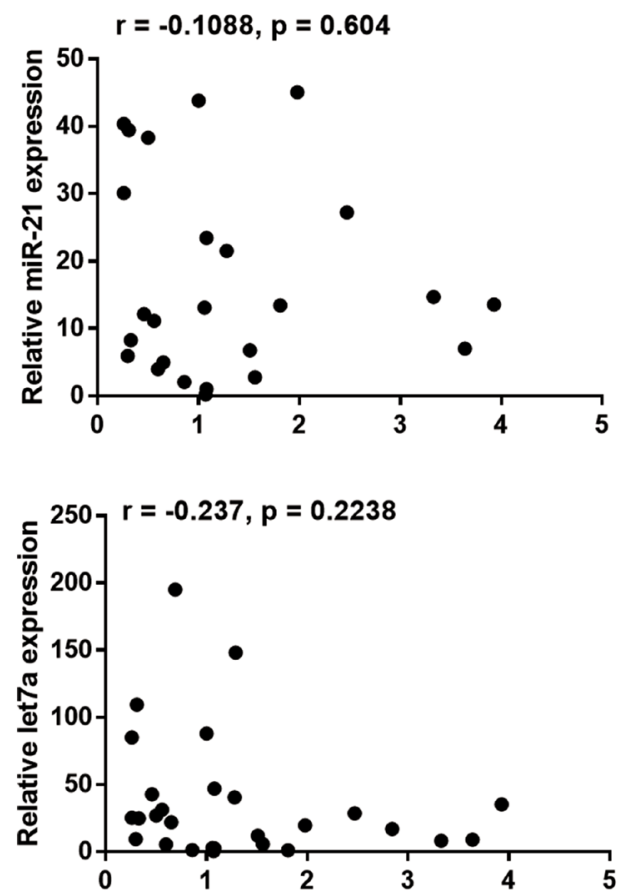

Glomerular Filtration Rate
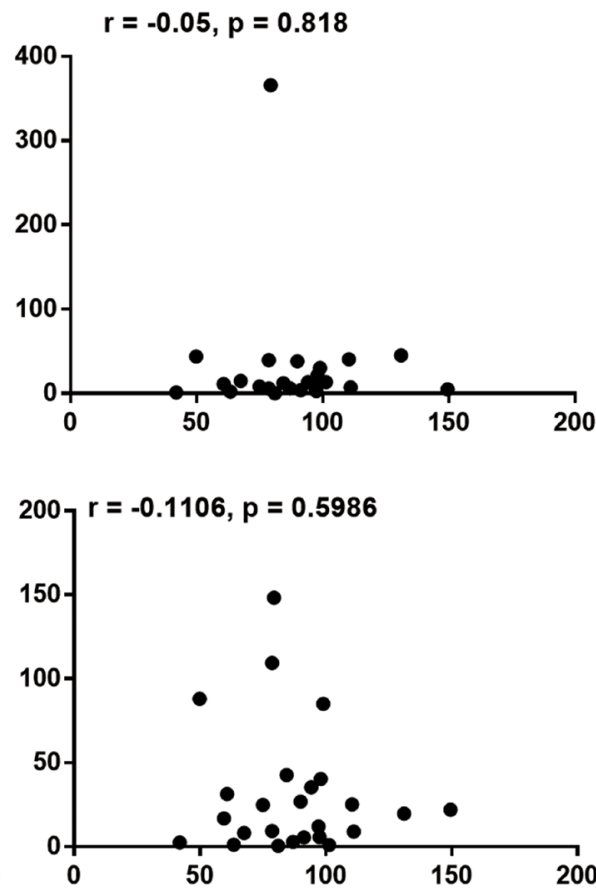

Serum Creatinine
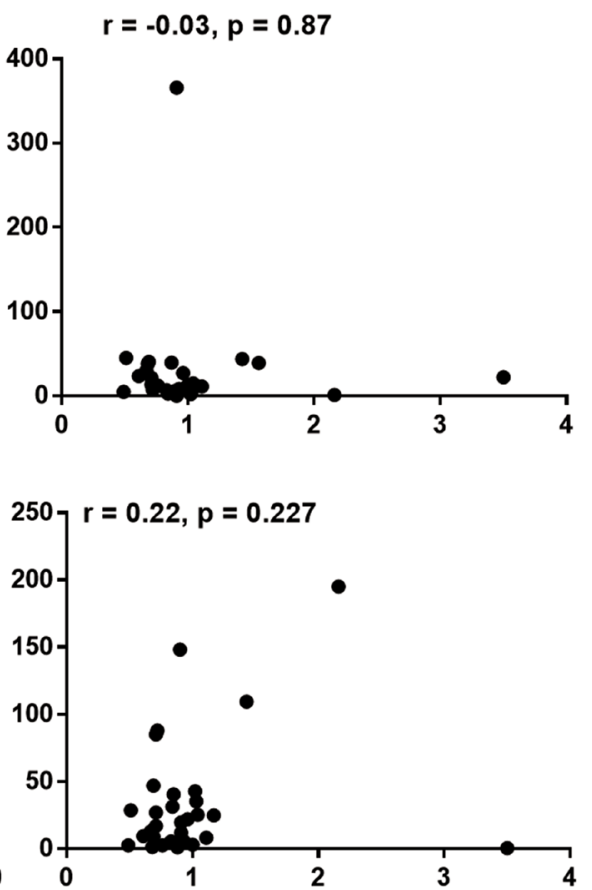

Figure 3. Correlation between expressions of urinary exosome-associated miRNAs and clinical biomarkers of kidney function. The graphs show correlation between relative miR-21, let-7a and miR-10a/b fold-change expression and urine protein leakage measuring through urine protein creatinine index (UPCI), glomerular filtration rate (GFR) and serum creatinine (sCr). The correlations were calculated using Pearson's correlation test. There are four missing points for UPCI, nine missing points for GFR, and three missing points for $\mathrm{sCr}$ as not all parameters were measured at all time points. A p-value less than 0.05 were the cut-off for statistically significant correlations. The $\mathrm{x}$-axis represents relative miRNA expression normalized with cel-39 spike-in control compare to active LN patients. 
Urine Protein Leakage
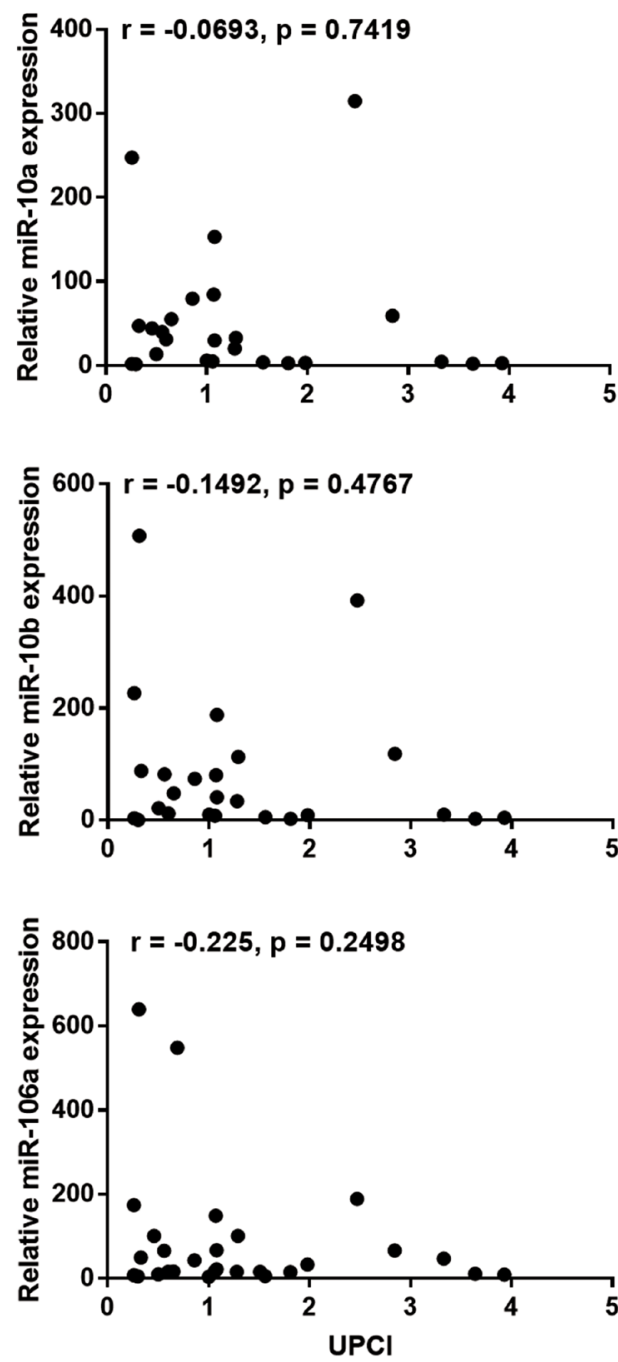

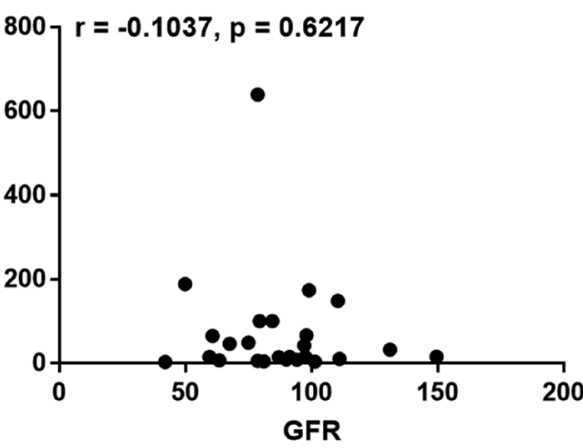

Serum Creatinine
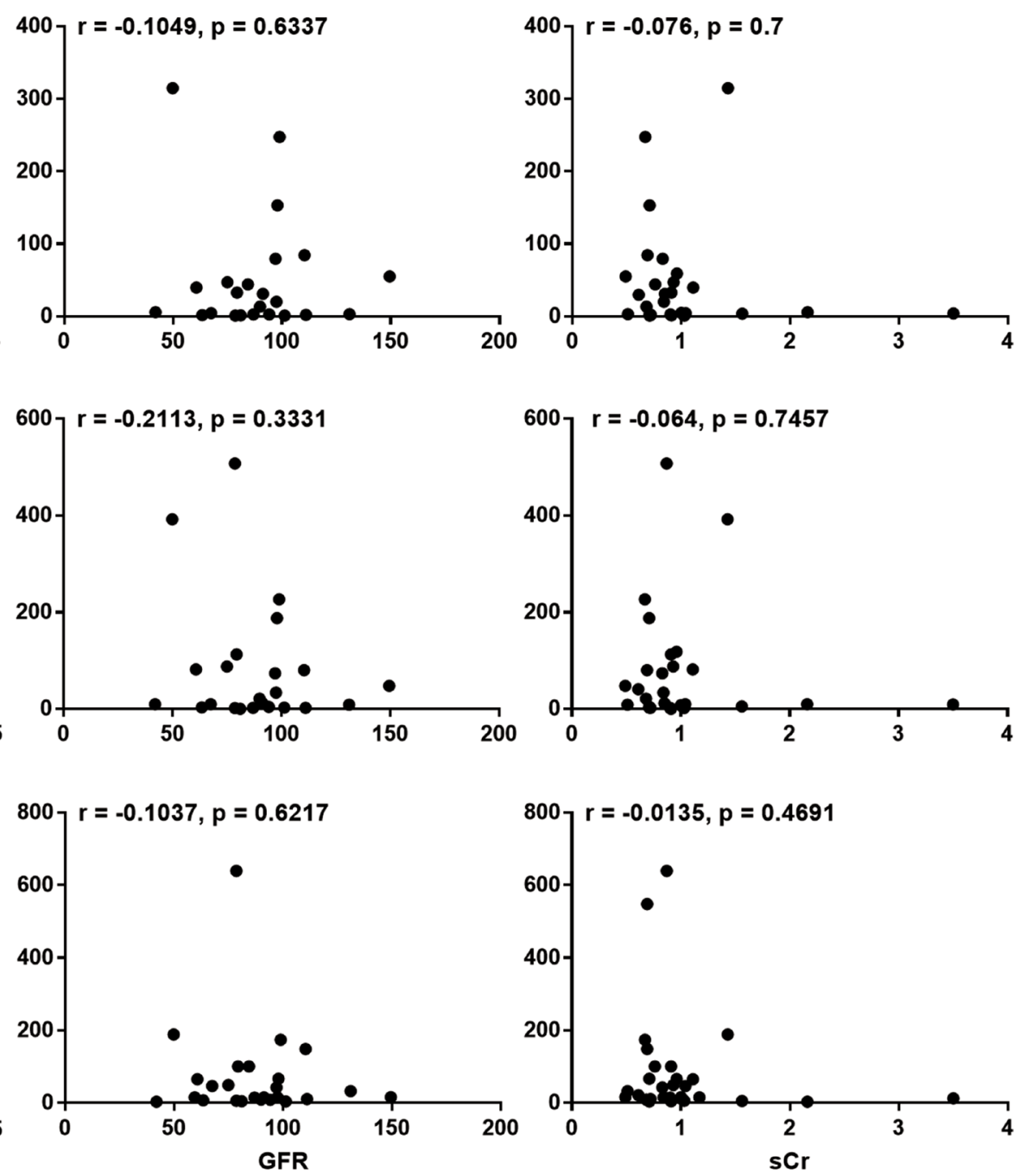

Figure 3. (Continued)

in random visits of active $(n=13)$ and inactive $(n=18) \mathrm{LN}$ patients. As we expected, let-7a and miR-21 were significantly down-regulated in urinary exosomes from patients with active LN, while miR-10a/b wasnot significantly different between the relapse and remission stages (Figure 2B). These results suggested that urinary exosomal miRNA might play an important role in LN during disease flare and might be able to represents disease stages mirroring prognosis biomarker.

In subsequent, we analyzed the correlation between urinary exosomal miRNA expression and kidney function (serum creatinine, urine protein leakage and glomerular filtration rate). Unexpectedly, none of the urinary exosome-associated miRNAs were significantly correlated with kidney function parameters (Figure 3). Even though the correlations were not significant, negative correlation was found in all miRNA expression with proteinuria and glomerular filtration rate. This might be implied that regulation of miRNA through exosome is able to control cells response and important in disease pathogenesis.

\section{Discussion}

In this report, we firstly characterized urinary exosome containing candidate miRNA expression (miR-21, miR-10a/b, and let-7a) in LN patients before treatment and after enter remission stage. In addition, we validated the results in random urines from active and inactive LN patients. Interestingly, our study found two miRNAs; let-7a and miR-21, were down-regulated in urinary exosomes during disease flare and down-regulation of let-7a was also consistent with our previous study. ${ }^{22}$ Because aberrant miRNA expression might lead to changing cell phenotypes and functions, down-regulation of both miRNAs might involve in defected renal cells functions in order to control inflammation.

For the let-7, it was one of the first discovered miRNAs in nematodes. ${ }^{27}$ In humans, there are several isoforms of the let-7 family sharing their "seed region" that are mostly characterized as tumor-suppressor miRNA. ${ }^{27}$ In spontaneous developed LN mouse model, experiments found that let-7a was able to control inflammation via inhibition of interleukin (IL)-6 expressions. ${ }^{28}$ Studies of kidney biopsies from patients with class IV LN revealed down-regulation of let-7a, which 
was also seen in mesangial cells stimulated with immune complexes. ${ }^{29}$ This might lead to over-production of IL- 6 in the kidneys of patients with active LN. ${ }^{30}$ Thus, down-regulation of let-7a may result in the loss of epigenetic regulatory systems within kidney. It is therefore interesting to study the epigenetic function of exosomes-containing let-7a in vivo. This might lead to new treatment target for patients with active LN to ameliorate disease fluctuations and its expression might be able to use as potential biomarkers to predict disease flare. However, the large sample sizes with different subset of autoimmune diseases and nephrotic syndrome patients are needed to confirm the specificity of let-7a.

Despite the miR-21 has been well characterized as a crucial factor in fibrogenesis and tissue repair in various organs, ${ }^{20,31}$ recent evidences showed that miR-21 also mediated anti-inflammatory effects in primary macrophages via the inhibition of IL-12 and PDCD4. ${ }^{32,33}$ The miR-21 has been shown to target several genes in the JAK-STAT pathway (CSF3R, SPRY2, IL23R, CNTFR, IL15, IL7, IL13RA1, STAT3, SOS2, SPRY1, PIK3R1, LIFR, IL9, JAK3, IL12A and IL13RA2) which are related to inflammatory processes. ${ }^{34}$ Thus, down-regulation of urinary exosomes containing miR-21 might represent the dysfunction of the inflammatory regulation. In contrast to let-7a, studies in kidney biopsies from patients with class IV LN and in cell-free urine showed an up-regulation of miR-21 expression. ${ }^{17,35}$ As miR-21 could be produced from various types of cells especially leukocytes, it is suspected that increasing of miR-21 in cell-free urine or kidney biopsy might be a consequent from leukocyte accumulation. Furthermore, increasing of urinary exosome containing miR-21 might be delivered from various kidney cells types. The further study to characterize specific cell types that contribute to the significant down-regulation of miR-21 in urine exosomes might be necessary to confirm this inconsistency.

We have previously reported that $\mathrm{miR}-10 \mathrm{a} / \mathrm{b}$ were the most abundant miRNA in mesangial cells and was significantly down-regulated in mesangial cells treated with immune complexes, resulting in increased IL-8 expression. ${ }^{22}$ Unlike let- $7 \mathrm{a}$, the level of $\mathrm{miR}-10 \mathrm{a} / \mathrm{b}$ in urinary exosomes was not statistically different between LN patients with active and inactive disease. The observation of exosome generation in each part of the nephron previously revealed production of exosomes by specific renal cell types including collecting duct intercalating cells, collecting duct principal cells, podocytes, and proximal tubule, thick ascending limb and thin descending limb epithelial cells, but not by mesangial cells. ${ }^{36}$ It is therefore possible that exosomes containing miR-10a/b from other cell types might affect the results and this might not be able to discriminate between active and inactive LN patients The study of miRNA in cell-specific urinary exosomes may provide a better understanding about urinary exosomal miRNA.

Previous results showed that urinary exosomal miR-29c showed a strong correlation between its expression and fibrosis occurring in SLE as analyzed by ROC curves. ${ }^{37}$ Although the correlations of our miRNA expression and kidney function were not reached statistically significant, this might be because those classical markers are not sensitive enough to define LN disease stage. Moreover, we hypothesize that the concentration of individual urine samples and treatment intervention may also affect miRNA expression. Another limitation might come from the variation due to the randomly collected urine in each individual LN patients. The standard miRNA expression normalization method in urinary exosome is also necessary to provide the consistent reports among researchers. Identifying the number of each renal cell type-specific urinary exosomes in LN patients and the number of EVs might be accounted for in miRNA expression normalization since similar urine volume could give different number of exosomes. However, the expression was not significant correlated with kidney function marker, we notice that most of miRNA expressions were negatively correlated with proteinuria and GFR. This raise the question whether exosomal miRNA might be associated with disease pathogenesis.

\section{Conclusions}

In conclusion, our study provides additional informationon miRNA expression within urinary exosomes isolated from patients with active and inactive LN. Let-7a and miR-21 were down-regulated in active LN patients, which might represent the expression of miRNA in the kidney and act as liquid biomarkers of present organ destruction. Furthermore, our results highlight that distinct miRNAs were selectively packed in urinary exosomes at different disease levels. This inferred that urinary exosomes containing miRNAs might be employed to regulate cellular responses in nephron systems. Thus, the benefit of urinary exosomes containing miRNA might be that they mirror the pathological response in the kidney which is ideal for a non-invasive biomarker.

\section{Acknowledgments}

The authors declare that there is no conflict of interest regarding the publication of this article. This study was supported by funding from the Royal Golden Jubilee PhD scholarship Grant (PhD660 program 2014, grant no. PHD/0321/ 2551), International Network for Lupus Research (grant no. IRN59W0004), the Thailand 661 Research Fund, International Research Integration, Chula Research Scholar, Ratchadaphiseksomphot Endowment Fund. We are grateful to Assist. Prof. Asada Leelahavanichkul, MD, PhD and Dr. Poorichaya Somparn who provided us advice regarded to exosomes detection and LN samples collection. We thank Simon Teteris, $\mathrm{PhD}$, from the Edanz Group (www.edanzediting.com/ac), for editing the English text of a draft of this manuscript.

\section{References}

1. Lech M, Anders HJ. The pathogenesis of lupus nephritis. J Am Soc Nephrol. 2013;24(9):1357-66.

2. Chan TM. Treatment of severe lupus nephritis: the new horizon. Nat Rev Nephrol. 2015;11(1):46-61.

3. Hahn BH, McMahon MA, Wilkinson A, Wallace WD, Daikh DI, Fitzgerald JD, et al. American College of Rheumatology guidelines for screening, treatment, and management of lupus nephritis. Arthritis Care Res (Hoboken). 2012;64(6):797-808.

4. Anders HJ, Weidenbusch M, Rovin B. Unmet medical needs in lupus nephritis: solutions through evidence-based, personalized medicine. Clin Kidney J. 2015;8(5):492-502.

5. Raposo G, Stoorvogel W. Extracellular vesicles: exosomes, microvesicles, and friends. J Cell Biol. 2013;200(4):373-83. 
6. S ELA, Mager I, Breakefield XO, Wood MJ. Extracellular vesicles: biology and emerging therapeutic opportunities. Nat Rev Drug Discov. 2013;12(5):347-57.

7. Maas SLN, Breakefield XO, Weaver AM. Extracellular Vesicles: Unique Intercellular Delivery Vehicles. Trends Cell Biol. 2017;27(3):172-88.

8. Robbins PD, Morelli AE. Regulation of immune responses by extracellular vesicles. Nat Rev Immunol. 2014;14(3):195-208.

9. Qian Z, Shen Q, Yang X, Qiu Y, Zhang W. The Role of Extracellular Vesicles: An Epigenetic View of the Cancer Microenvironment. Biomed Res Int. 2015;2015:649161.

10. Dear JW, Street JM, Bailey MA. Urinary exosomes: a reservoir for biomarker discovery and potential mediators of intrarenal signalling. Proteomics. 2013;13(10-11):1572-80.

11. Li JY, Yong TY, Michael MZ, Gleadle JM. Review: The role of microRNAs in kidney disease. Nephrology (Carlton). 2010;15(6):599-608.

12. Mulcahy LA, Pink RC, Carter DR. Routes and mechanisms of extracellular vesicle uptake. J Extracell Vesicles. 2014;3.

13. Ichii $\mathrm{O}$, Ohta $\mathrm{H}$, Horino $\mathrm{T}$, Nakamura $\mathrm{T}$, Hosotani $\mathrm{M}$, Mizoguchi $\mathrm{T}$, et al. Urinary exosome-derived microRNAs reflecting the changes of renal function and histopathology in dogs. Sci Rep. 2017;7:40340.

14. Perez-Hernandez J, Forner MJ, Pinto C, Chaves FJ, Cortes R, Redon J. Increased Urinary Exosomal MicroRNAs in Patients with Systemic Lupus Erythematosus. PLoS One. 2015;10(9):e0138618.

15. Ichii O, Otsuka-Kanazawa S, Horino T, Kimura J, Nakamura T, Matsumoto $\mathrm{M}$, et al. Decreased miR-26a expression correlates with the progression of podocyte injury in autoimmune glomerulonephritis. PLoS One. 2014; 9(10):e110383.

16. Lv LL, Cao YH, Ni HF, Xu M, Liu D, Liu H, et al. MicroRNA-29c in urinary exosome/microvesicle as a biomarker of renal fibrosis. Am J Physiol Renal Physiol. 2013;305(8):F1220-7.

17. Chafin CB, Regna NL, Hammond SE, Reilly CM. Cellular and urinary microRNA alterations in NZB/W mice with hydroxychloroquine or prednisone treatment. Int Immunopharmacol. 2013;17(3):894-906.

18. Chafin CB, Reilly CM. MicroRNAs implicated in the immunopathogenesis of lupus nephritis. Clin Dev Immunol. 2013;2013:430239.

19. Wang S, Wan X, Ruan Q. The MicroRNA-21 in Autoimmune Diseases. Int J Mol Sci. 2016;17(6).

20. Chau BN, Xin C, Hartner J, Ren S, Castano AP, Linn G, et al. MicroRNA-21 promotes fibrosis of the kidney by silencing metabolic pathways. Sci Transl Med. 2012;4(121):121ra18.

21. Denby L, Ramdas V, McBride MW, Wang J, Robinson H, McClure J, et al. miR-21 and miR-214 are consistently modulated during renal injury in rodent models. Am J Pathol. 2011;179(2):661-72.

22. Tangtanatakul P, Thammasate B, Jacquet A, Reantragoon R, Pisitkun T, Avihingsanon Y, et al. Transcriptomic profiling in human mesangial cells using patient-derived lupus autoantibodies identified miR-10a as a potential regulator of IL8. Sci Rep. 2017;7(1):14517.
23. Wang N, Zhou Y, Jiang L, Li D, Yang J, Zhang CY, et al. Urinary microRNA -10a and microRNA-30d serve as novel, sensitive and specific biomarkers for kidney injury. PLoS One. 2012;7(12):e51140.

24. Meng F, Henson R, Wehbe-Janek H, Smith H, Ueno Y, Patel T. The MicroRNA let-7a modulates interleukin-6-dependent STAT-3 survival signaling in malignant human cholangiocytes. J Biol Chem. 2007;282(11): 8256-64.

25. Weening JJ, D’Agati VD, Schwartz MM, Seshan SV, Alpers CE, Appe $\mathrm{GB}$, et al. The classification of glomerulonephritis in systemic lupus erythematosus revisited. J Am Soc Nephrol. 2004;15(2):241-50.

26. Witwer KW, Buzas EI, Bemis LT, Bora A, Lasser C, Lotvall J, et al Standardization of sample collection, isolation and analysis methods in extracellular vesicle research. J Extracell Vesicles. 2013;2.

27. Lee H, Han S, Kwon CS, Lee D. Biogenesis and regulation of the let-7 miRNAs and their functional implications. Protein Cell. 2016;7(2):100-13.

28. Sung SY, Liao CH, Wu HP, Hsiao WC, Wu IH, Jinpu, et al. Loss of let-7 microRNA upregulates IL-6 in bone marrow-derived mesenchymal stem cells triggering a reactive stromal response to prostate cancer. PLoS One. 2013;8(8):e71637.

29. Costa-Reis P, Russo PA, Zhang Z, Colonna L, Maurer K, Gallucci S, et al The Role of MicroRNAs and Human Epidermal Growth Factor Receptor 2 in Proliferative Lupus Nephritis. Arthritis Rheumatol. 2015;67(9):2415-26.

30. Herrera-Esparza R, Barbosa-Cisneros O, Villalobos-Hurtado R, Avalos -Diaz E. Renal expression of IL-6 and TNFalpha genes in lupus nephritis. Lupus. 1998;7(3):154-8.

31. Dey N, Ghosh-Choudhury N, Kasinath BS, Choudhury GG. TGFbeta -stimulated microRNA-21 utilizes PTEN to orchestrate AKT/mTORC1 signaling for mesangial cell hypertrophy and matrix expansion. PLoS One. 2012;7(8):e42316.

32. Asangani IA, Rasheed SA, Nikolova DA, Leupold JH, Colburn NH, Post S et al. MicroRNA-21 (miR-21) post-transcriptionally downregulates tumor suppressor Pdcd4 and stimulates invasion, intravasation and metastasis in colorectal cancer. Oncogene. 2008;27(15):2128-36.

33. Sheedy FJ. Turning 21: Induction of miR-21 as a Key Switch in the Inflammatory Response. Front Immunol. 2015;6:19.

34. Li HW, Xie Y, Li F, Sun GC, Chen Z, Zeng HS. Effect of miR-19a and miR-21 on the JAK/STAT signaling pathway in the peripheral blood mononuclear cells of patients with systemic juvenile idiopathic arthritis. Exp Ther Med. 2016;11(6):2531-6.

35. Krasoudaki E, Banos A, Stagakis E, Loupasakis K, Drakos E, Sinatkas $\mathrm{V}$, et al. Micro-RNA analysis of renal biopsies in human lupus nephritis demonstrates up-regulated miR-422a driving reduction of kallikrein -related peptidase 4. Nephrol Dial Transplant. 2016;31(10):1676-86.

36. Pomatto MAC, Gai C, Bussolati B, Camussi G. Extracellular Vesicles in Renal Pathophysiology. Front Mol Biosci. 2017;4:37.

37. Sole C, Cortes-Hernandez J, Felip ML, Vidal M, Ordi-Ros J. miR-29c in urinary exosomes as predictor of early renal fibrosis in lupus nephritis. Nephrol Dial Transplant. 2015;30(9):1488-96. 